\title{
Achieving a physiological cortisol profile with once-daily dual-release hydrocortisone: a pharmacokinetic study
}

\author{
Gudmundur Johannsson', Hans Lennernäs ${ }^{2}$, Claudio Marelli³, Kevin Rockich ${ }^{4}$ \\ and Stanko Skrtic ${ }^{1,5}$ \\ 1'Department of Endocrinology, Institute of Medicine, Sahlgrenska Academy, University of Gothenburg, \\ Gothenburg, Sweden, ²Department of Pharmacy, Uppsala University, Uppsala, Sweden, \\ ${ }^{3}$ Shire International GmbH, Zug, Switzerland, ${ }^{4}$ Shire PLC, Wayne, Pennsylvania, USA and \\ ${ }^{5}$ AstraZeneca R\&D, Mölndal, Sweden
}

\author{
Correspondence \\ should be addressed \\ to G Johannsson \\ Email \\ gudmundur.johannsson@ \\ medic.gu.se
}

\begin{abstract}
Objective: Oral once-daily dual-release hydrocortisone (DR-HC) replacement therapy was developed to provide a cortisol exposure-time profile that closely resembles the physiological cortisol profile. This study aimed to characterize single-dose pharmacokinetics (PK) of DR-HC 5-20 mg and assess intrasubject variability.

Methods: Thirty-one healthy Japanese or non-Hispanic Caucasian volunteers aged 20-55 years participated in this randomized, open-label, PK study. Single doses of DR-HC 5, $15(3 \times 5)$, and $20 \mathrm{mg}$ were administered orally after an overnight fast and suppression of endogenous cortisol secretion. After estimating the endogenous cortisol profile, PK of DR-HC over $24 \mathrm{~h}$ were evaluated to assess dose proportionality and impact of ethnicity. Plasma cortisol concentrations were analyzed using liquid chromatography-tandem mass spectrometry. PK parameters were calculated from individual cortisol concentration-time profiles.

Results: DR-HC $20 \mathrm{mg}$ provided higher than endogenous cortisol plasma concentrations $0-4 \mathrm{~h}$ post-dose but similar concentrations later in the profile. Cortisol concentrations and PK exposure parameters increased with increasing doses. Mean maximal serum concentration $\left(\mathrm{C}_{\max }\right)$ was 82.0 and $178.1 \mathrm{ng} / \mathrm{mL}$, while mean area under the concentration-time curve (AUC) $0_{-\infty}$ was 562.8 and $1180.8 \mathrm{~h} \times \mathrm{ng} / \mathrm{mL}$ with DR-HC 5 and $20 \mathrm{mg}$ respectively. Withinsubject PK variability was low (<15\%) for DR-HC $20 \mathrm{mg}$. All exposure PK parameters were less than dose proportional (slope <1). PK differences between ethnicities were explained by body weight differences.

Conclusions: DR-HC replacement resembles the daily normal cortisol profile. Within-subject day-to-day PK variability was low, underpinning the safety of DR-HC for replacement therapy. DR-HC PK were less than dose proportional - an important consideration when managing intercurrent illness in patients with adrenal insufficiency.
\end{abstract}

European Journal of

Endocrinology

(2016) 175, 85-93

\section{Introduction}

Adrenal insufficiency (AI) is a life-threatening orphan disease that requires daily glucocorticoid replacement to increase cortisol concentrations to pre-disease values $(1,2)$. Hydrocortisone is the most commonly used glucocorticoid replacement therapy and requires multiple daily oral administrations to provide an adequate cortisol concentration-time profile (1). In a typical dosing regimen, hydrocortisone is taken two or three times per day, with the highest dose in the morning, a lower dose at midday and, if necessary, a third dose in the late afternoon or early evening (3). This conventional treatment regimen has demonstrated efficacy in AI but long-term clinical www.eje-online.org

DOI: 10.1530/EJE-15-1212 (c) 2016 The authors Published by Bioscientifica Ltd.

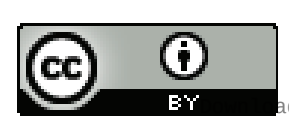

This work is licensed under a Creative Commons Attribution 3.0 Unported License 
outcomes remain unsatisfactory, with higher premature mortality and morbidity and impaired quality of life (QoL) compared with the general population $(4,5,6,7,8)$. Large studies have shown that the all-cause mortality risk is more than two-fold higher in patients with primary $\mathrm{AI}$ than in the general population, mainly resulting from cardiovascular, malignant, and infectious diseases $(4,5)$. Furthermore, patients with chronic AI often experience adrenal crisis despite treatment and education, with an associated mortality rate of $6.3 \%$ (9).

The inability of conventional glucocorticoid replacement to provide a plasma cortisol exposure-time profile that resembles the circadian physiological cortisol profile is likely to have direct adverse effects on metabolism, general health, and QoL $(1,3)$. Higher than physiological cortisol exposure in the late afternoon and evening may be a particularly important cause of metabolic dysfunction and altered sleep patterns, but excessive total glucocorticoid exposure and inadequate rescue therapy during intercurrent illness will also have important clinical implications, including increased morbidity and mortality related to infectious disease and increased cardiometabolic risk $(1,3,6,9,10,11,12,13$, 14). In a study in hypopituitary patients, glucocorticoid replacement therapy was associated with dose-related increases in BMI, triglycerides, low-density lipoprotein cholesterol, and total cholesterol (12). Abnormally high cortisol levels in the evening have been associated with insomnia and increased sleep disturbance $(11,14)$.

Modern biopharmaceutical techniques have allowed the development of a once-daily, dual-release hydrocortisone (DR-HC) tablet, comprising an immediaterelease coating and an extended-release core, for oral glucocorticoid replacement therapy in AI (15). This dualrelease formulation was developed to provide a cortisol exposure-time profile that more closely resembles the physiological serum cortisol profile than provided by standard formulations (16). Clinical trials in healthy volunteers and patients with AI have demonstrated that DR-HC produces a diurnal plasma cortisol profile that mimics the daytime circadian-based physiological serum cortisol profile more closely than conventional glucocorticoid replacement therapy with immediaterelease tablets $(15,16)$. The study conducted in patients with AI also showed that treatment with once-daily DR-HC tablets significantly improved metabolic factors and QoL compared with conventional hydrocortisone tablets administered three times per day (16). Furthermore, a prospective trial in patients with AI showed that DR-HC significantly reduced BMI and hemoglobin A1c
(HbA1c) and stabilized QoL compared with conventional hydrocortisone therapy (17).

This study was conducted to expand the pharmacokinetic (PK) data of DR-HC to provide support for dosing recommendations. Hence, the main study objectives were to characterize the single-dose plasma PK of DR-HC tablets across the dose range of 5-20 mg in the fasted state in healthy volunteers and to assess intrasubject variability.

\section{Subjects and methods}

\section{Subjects}

Healthy men and women aged 20-55 years with a BMI $18-30 \mathrm{~kg} / \mathrm{m}^{2}$, body weight of $\geq 50 \mathrm{~kg}$ for men and $\geq 45 \mathrm{~kg}$ for women, and who were of either Japanese descent or non-Hispanic Caucasian were included in the study. Exclusion criteria included: any known metabolic or endocrine disorders; clinical or laboratory signs of significant cerebral, cardiovascular, respiratory, renal, hepatobiliary, pancreatic, or gastrointestinal emptying/ motility disease that could interfere with study assessments or completion; history of hydrocortisone intolerance; hypersensitivity to DR-HC, dexamethasone, or any of their excipients; systemic fungal infection; hepatitis B or $\mathrm{C}$ infection; known immune deficiency virus; taken any investigational study drug within 30 days of starting the study; drug or alcohol abuse within 1 year of starting the study. Any agent that may interfere with hydrocortisone PK or hepatic drug metabolizing capacity within 14 days before starting the study were not permitted. Pregnant or lactating women were not eligible for the trial.

All volunteers provided written informed consent before entering the study. The local Ethics Committee approved the study protocol and the study was performed according to the principles of Good Clinical Practice and the Declaration of Helsinki. The trial was conducted between 15 November 2013 and 21 December 2013.

\section{Study design}

This was a randomized, open-label, four-period crossover, single-dose PK study of oral DR-HC tablets conducted at a single center. All subjects were screened within 21 days before the first treatment period and had a baseline $24-\mathrm{h}$ assessment of endogenous cortisol secretion, performed after overnight fast and before the first treatment period. Eligible subjects were then randomized to one of four treatment sequences, each comprising four 3-day 
treatment periods separated by wash-out periods of at least $72 \mathrm{~h}$. Blood samples for PK assessments were collected at $15,30,45,60,90,120$, and $150 \mathrm{~min}$, hourly from 3 to $10 \mathrm{~h}$, and at 12,15 , and $24 \mathrm{~h}$ after dosing with study drug in order to provide evaluable concentration-time profiles. Subjects were provided with standardized meals throughout the study.

Medical history, physical examination, vital signs, and medication history were assessed at baseline. Subjects were monitored throughout the study for adverse events (AEs). Subjects returned to the clinical trial unit approximately 1 week after the final dose for a follow-up assessment, which included a physical examination, vital signs, ECG, and routine laboratory parameters.

\section{Interventions}

Oral dexamethasone $1 \mathrm{mg}$ was administered at 18:00 and $23: 00 \mathrm{~h}( \pm 15 \mathrm{~min})$ on day 1 and at 07:00, 11:00, $17: 00$, and $23: 00 \mathrm{~h}( \pm 15 \mathrm{~min})$ on day 2 of each treatment period to suppress endogenous cortisol secretion during PK sampling (18). Endogenous cortisol suppression was defined as a pre-dose plasma cortisol concentration $\leq 50 \mathrm{nmol} / \mathrm{L}$ (within $15 \mathrm{~min}$ before 08:00 h on day 2).

Single doses of DR-HC produced at different manufacturing sites (Recipharm, Stockholm, Sweden (test site) and Galenica, Malmö, Sweden (reference site)) were evaluated for bioequivalence. Doses of 5, $15(3 \times 5)$, and $20 \mathrm{mg}$ (test site) and $20 \mathrm{mg}$ (reference site) were administered orally once daily at $08: 00 \mathrm{~h}$ on day 2 of the treatment period after overnight fast. Assuming that bioequivalence of DR-HC manufactured at the different sites is established, administration of the two $20 \mathrm{mg}$ doses of DR-HC enabled the evaluation of intrasubject variability in PK.

\section{Analytical methods}

Plasma cortisol concentrations were analyzed using a validated liquid chromatography-tandem mass spectrometry (LC-MS/MS) method with an assay range of $1-500 \mathrm{ng} / \mathrm{mL}$ (Quotient Bio Analytical Sciences, Cambridgeshire, UK). The lower limit of quantification (LLOQ) was $1 \mathrm{ng} / \mathrm{mL}$ and the precision of the assay (coefficient of variation) was $\leq 5.7 \%$.

\section{Statistical analysis}

All PK analyses were performed on the PK population, which included all subjects with at least one evaluable concentration-time profile. There were two definitions for an evaluable concentration-time profile: protocol defined and PK analysis plan (PKAP) defined, the latter being more conservative. Both definitions of an evaluable concentration-time profile included the following criteria: $>50 \%$ of samples with values above the LLOQ, a pre-dose plasma cortisol concentration $\leq 50 \mathrm{nmol} / \mathrm{L}$ (indicating dexamethasone suppression), and no major deviations related to investigational product intake (e.g. vomiting) or PK sampling. The PKAP-defined evaluable PK profile also had to have a good estimate of the terminal elimination phase, that is, an adjusted $R^{2}$ goodness-of-fit statistic $>80 \%$, and sufficient data to estimate the area under the concentration-time curve (AUC) with $\leq 10 \%$ of the integrand extrapolated. Data for the protocol-defined evaluable concentration-time profiles are primarily presented here. Demographics and baseline characteristics were summarized for the intentto-treat (ITT) population, which included all subjects who received the study drug.

PK analyses for hydrocortisone (cortisol) were determined by non-compartmental analysis using WinNonlin version 6.2 or higher (Pharsight Corp., St Louis, MO, USA). PK parameters were calculated from individual cortisol concentration-time profiles and individual baseline-corrected cortisol concentration-time profiles. The baseline-corrected concentration was calculated by subtracting the baseline value (measured within 15 min before treatment) from each concentration in each individual plasma concentration-time profile.

Allplasma AUC parameterswerecalculatedusinglinear/ logarithmic trapezoidal method. $\mathrm{AUC}_{0-\infty}$ was calculated by extrapolating the curve to infinity using the sum of $\mathrm{AUC}_{\text {last }}+\mathrm{C}_{\text {last }} / \lambda_{\mathrm{z}}$, where $\mathrm{AUC}_{\text {last }}$ is the AUC through to the last measurable concentration, $\mathrm{C}_{\text {last }}$ is the last measurable concentration, and $\lambda_{\mathrm{z}}$ is the terminal rate determined using goodness-of-fit statistics and an adequate number of points in the terminal phase of the curve. $\mathrm{AUC}_{0-12 \mathrm{~h}}$ was calculated from time zero to the sample taken at $12 \mathrm{~h}$ after dosing. The terminal half-life was obtained from the elimination rate constant as $\ln 2 / \lambda_{\mathrm{z}}$. Summary statistics were determined for all PK parameters by treatment and for the endogenous cortisol plasma assessment at each time point.

Bioequivalence, defined as the $90 \%$ confidence interval (CI) being within the 80-125\% limits, was determined for the two $20 \mathrm{mg}$ tablets (from the test and reference sites) using the average bioequivalence approach (two one-sided tests procedure) $(19,20)$. Within-subject variability was examined with log-transformed maximal 
cortisol plasma concentration $\left(\mathrm{C}_{\max }\right), \mathrm{AUC}_{0-12 \mathrm{~h}}$, and $\mathrm{AUC}_{\text {last }}$ (uncorrected for pre-dose baseline cortisol concentrations). Dose proportionality of baseline-corrected and uncorrected PK parameters over the administered dose range (test site) was examined using the power model method, with ethnicity as a potential covariate.

\section{Results}

\section{Baseline characteristics}

Thirty-one patients were randomized and included in the ITT and PK analysis populations. Thirty subjects (97\%) completed the study; one subject withdrew for personal reasons after receiving two doses. The mean age of the study subjects was 38.5 years (range, 22-54 years), mean body weight was $69.3 \mathrm{~kg}$ (range, $48.8-84.7 \mathrm{~kg}$ ), mean BMI was $23.7 \mathrm{~kg} / \mathrm{m}^{2}$ for men and $21.9 \mathrm{~kg} / \mathrm{m}^{2}$ for women, $51.6 \%$ were of Japanese descent and $48.4 \%$ were Caucasian (Table 1). The Japanese and Caucasian groups were well matched for age, BMI, and sex, but the Japanese group had a lower mean (s.D.) weight of 66.5 (9.7) kg compared with the Caucasian group of $72.2(9.6) \mathrm{kg}$ due to two outliers in the Japanese group with very low weights (48.8 and $52.4 \mathrm{~kg}$ ).

More subjects had protocol- versus PKAP-defined evaluable concentration-time profiles. For uncorrected PK parameters with the two $20 \mathrm{mg}$ tablets, 23 (77\%) and 19 (63\%) subjects had evaluable PK profiles using the PKAP definition, while 29 (97\%) and 30 (100\%) subjects were evaluable using the protocol definition. The difference between the two populations was the inclusion of subjects that had a poorly estimated $\lambda_{z}$, as indicated by an adjusted $R^{2}$ of $\leq 0.8$, due to the presence of endogenous

Table 1 Demographic and baseline characteristics: ITT population. Results are presented as $n(\%)$ for categorical variables and mean (s.D.) for continuous variables.

\begin{tabular}{lc}
\hline & ITT $(n=31)$ \\
\hline Age (years) & $38.5(10.1)$ \\
Sex & $26(83.9 \%)$ \\
Male & $5(16.1 \%)$ \\
Female & $69.3(9.9)$ \\
Body weight $(\mathrm{kg})$ & \\
BMI $\left(\mathrm{kg} / \mathrm{m}^{2}\right)$ & $23.7(1.7)$ \\
Male & $21.9(2.6)$ \\
Female & $15(48.4 \%)$ \\
Race & $16(51.6 \%)$ \\
$\quad$ Non-Hispanic Caucasian & $10(32.3 \%)$ \\
$\quad$ Japanese & \\
Clinically relevant disease & \\
\hline
\end{tabular}

ITT, intent-to-treat. cortisol concentrations and the inability to fully correct for changing levels throughout the profile.

\section{Comparison of replacement hydrocortisone with endogenous cortisol profile}

Oral replacement treatment with DR-HC $20 \mathrm{mg}$ seemed to provide higher than endogenous cortisol plasma concentrations $0-4 \mathrm{~h}$ post-dose, with the peak concentration measured at $0.5 \mathrm{~h}$ after dosing, but similar plasma concentrations later in the profile, particularly from midday to the last evaluations in the day at 23:00h (after DR-HC administration) and midnight (endogenous cortisol) (Fig. 1). There was considerable intersubject variability in both the endogenous cortisol plasma profile and the cortisol plasma concentrations following dosing with DR-HC. For the first measurement of endogenous cortisol concentration at $06: 00 \mathrm{~h}$ on day 1 , values ranged between 20.4 and $190.0 \mathrm{ng} / \mathrm{mL}$, while midnight levels ranged between 9.6 and $105.0 \mathrm{ng} / \mathrm{mL}$ and levels at 08:00 h on day 2 ranged from 53.1 to $194.0 \mathrm{ng} / \mathrm{mL}$; all subjects achieved peak plasma concentrations of $\geq 95 \mathrm{ng} / \mathrm{mL}$ during the 24 -h evaluation of endogenous cortisol. Peak cortisol plasma concentrations $30 \mathrm{~min}$ after administration of DR-HC $20 \mathrm{mg}$ (test site) ranged from 120.0 to $266.0 \mathrm{ng} / \mathrm{mL}$, while at $23: 00 \mathrm{~h}$ concentrations had diminished to $0-65.0 \mathrm{ng} / \mathrm{mL}$. AUC last following dosing with DR-HC $20 \mathrm{mg}$ (test site) ranged from 836.3 to $1670.9 \mathrm{~h} \times \mathrm{ng} / \mathrm{mL}$.

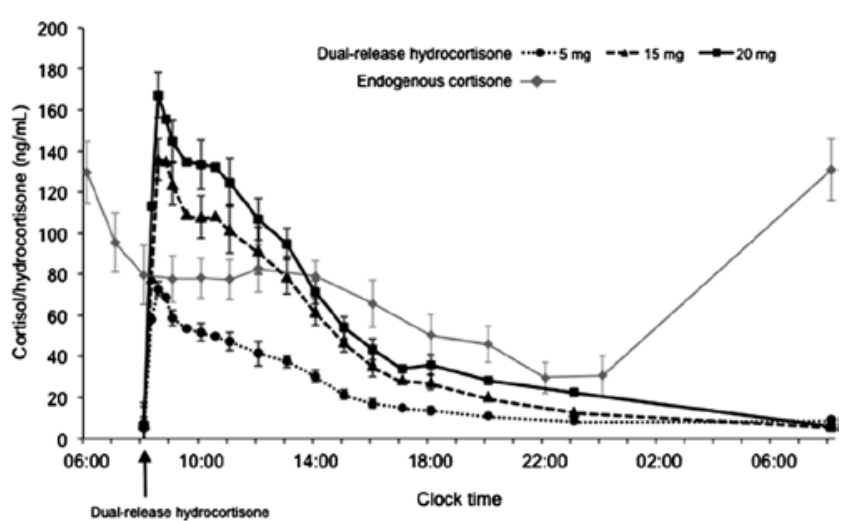

Figure 1

Mean $(95 \% \mathrm{Cl})$ plasma concentration-time profiles for endogenous cortisol and after single oral doses of DR-HC 5, 15 , and $20 \mathrm{mg}$ (test site) in healthy subjects in the PK population. Endogenous cortisol concentrations were not assessed after 00:00 until 06:00 on day 2 . 
Table 2 Plasma PK variables for DR-HC in healthy subjects in the PK population. All pharmacokinetic variables are uncorrected and presented as mean (s.D.), with the exception of $\mathrm{T}_{\max }$, which is presented as median (range).

\begin{tabular}{l} 
Parameter \\
\hline $\mathrm{C}_{\max }(\mathrm{ng} / \mathrm{mL})$ \\
$\mathrm{AUC}_{0-\infty}(\mathrm{h} \times \mathrm{ng} / \mathrm{mL})$ \\
$\mathrm{AUC}_{0-12 \mathrm{~h}}(\mathrm{~h} \times \mathrm{ng} / \mathrm{mL})$ \\
$\mathrm{T}_{\max }(\mathrm{h})$ \\
Terminal half-life $(\mathrm{h})$ \\
$\%$ extrapolated AUC $(\%)^{c}$ \\
\hline
\end{tabular}

\begin{tabular}{c}
\hline $\mathbf{5} \mathbf{~ m}^{\mathbf{a}}$ \\
\hline $82.0(18.2)$ \\
$562.8(141.0)$ \\
$371.8(75.8)$ \\
$0.5(0.3-1.0)$ \\
$13.7(8.0)$ \\
$17.4(10.8)$ \\
\hline
\end{tabular}

\begin{tabular}{c}
\hline${\mathbf{1 5 ~} \mathbf{~ g}^{\mathbf{a}}}$ \\
\hline $148.8(29.3)$ \\
$991.6(162.0)$ \\
$770.4(209.0)$ \\
$0.5(0.3-24.0)$ \\
$8.3(5.1)$ \\
$7.5(6.7)$ \\
\hline
\end{tabular}

\begin{tabular}{c}
\hline $\mathbf{2 0} \mathbf{m g}^{\mathbf{a}}$ \\
\hline $177.1(25.5)$ \\
$1180.8(213.8)$ \\
$947.7(174.2)$ \\
$0.5(0.3-5.0)$ \\
$6.0(2.9)$ \\
$4.3(2.6)$ \\
\hline
\end{tabular}

\begin{tabular}{c}
\hline $\mathbf{2 0} \mathbf{m g}^{\mathbf{b}}$ \\
\hline $178.0(28.1)$ \\
$1162.1(175.7)$ \\
$919.5(169.7)$ \\
$0.5(0.3-5.0)$ \\
$6.7(3.6)$ \\
$5.7(4.9)$ \\
\hline
\end{tabular}

aTest site; ${ }^{b}$ reference site; 'percentage of the AUC resulting from extrapolation after the last measurable concentration. AUC, area under the concentration-time curve; $\mathrm{AUC}_{0-\infty}$, total area under the concentration-time curve; $\mathrm{AUC}_{0-12 \mathrm{~h}}$, area under the concentration-time curve to the last sample taken at $12 \mathrm{~h}$ after dosing; $\mathrm{C}_{\max }$ maximal serum concentration; $\mathrm{PK}$, pharmacokinetic.

\section{Plasma PK parameters for DR-HC}

Cortisol plasma concentrations and hydrocortisone PK exposure parameters increased with increasing doses of DR-HC, and a biphasic release profile was observed (Fig. 1 and Table 2). Plasma cortisol/hydrocortisone concentrations remained above baseline levels for at least $15 \mathrm{~h}$ after all doses. The percentage of exposure extrapolated in the plasma concentration-time profile (\% extrapolated AUC) was low (<6\%) for both $20 \mathrm{mg}$ doses. Mean PK parameters appeared to be similar if corrected or uncorrected for the pre-dose baseline cortisol concentration and when the PKAP definition for evaluable PK profiles was used.

Bioequivalence was demonstrated for the DR-HC $20 \mathrm{mg}$ tablets from the test and reference sites for corrected and uncorrected PK parameters, and for both definitions used for evaluable PK profiles (data not shown). Within-subject variability, determined for the two $20 \mathrm{mg}$ tablets from different manufacturing sites, was low and below 15\% for all examined PK parameters (Table 3).

\section{Dose proportionality}

All of the exposure PK parameters for cortisol examined (uncorrected and baseline corrected) were found to be less than dose proportional, with a slope $<1$, in the
5-20 mg dose range (Table 2). The dose-proportionality slopes for baseline-corrected PK parameters (90\% CI) were as follows: $\mathrm{C}_{\max }, 0.59 \quad(0.52-0.66) \mathrm{h} \times \mathrm{ng} / \mathrm{mL}$; $\mathrm{AUC}_{0-12 \mathrm{~h}}, 0.74(0.58-0.89) \mathrm{h} \times \mathrm{ng} / \mathrm{mL}$; and $\mathrm{AUC}_{\text {last }}, 0.79$ $(0.71-0.87) \mathrm{h} \times \mathrm{ng} / \mathrm{mL}$. The slope $(90 \% \mathrm{CI})$ for $\mathrm{AUC}_{0-\infty}$, which was only calculated for PKAP-defined evaluable concentration-time profiles due to the requirement for an adjusted $R^{2}>80 \%$, was 0.78 (90\% CI: $0.70-0.85$ ). Mean plasma $\mathrm{AUC}_{0-\infty}$ increased 1.8- and 2.1-fold, and $\mathrm{C}_{\max }$ increased 1.8- and 2.2-fold when the dose of DR-HC increased from 5 to $15 \mathrm{mg}$ and $20 \mathrm{mg}$ respectively. There were no differences between the two ethnic groups.

\section{Impact of ethnicity on PK parameters for DR-HC}

DR-HC PK parameters were similar for Caucasian and Japanese subjects, with the exception of two outliers, who were both Japanese females and had the lowest body weights recorded among enrolled subjects (48.8 and $52.4 \mathrm{~kg}$ ). Using the two one-sided tests procedure, a significant difference in $\mathrm{C}_{\max }$ due to ethnicity (group fixed-effect $P=0.0126$ (uncorrected) and $P=0.0103$ (corrected)) was found but, when subject body weight was added to the model, ethnicity lost significance indicating that the between-group differences in PK parameters were explained by differences in body weight.

Table 3 Within-subject variability for the two DR-HC $20 \mathrm{mg}$ tablets (reference and test sites) in the PK population.

\begin{tabular}{|c|c|c|c|c|c|}
\hline \multirow[b]{2}{*}{ Parameter } & \multicolumn{2}{|c|}{ Geometric LSMs } & \multirow[b]{2}{*}{ Within-subject \%CV } & \multirow[b]{2}{*}{ LSM ratio (test/reference) } & \multirow[b]{2}{*}{$90 \% \mathrm{Cl}$} \\
\hline & 20 mg (reference site) & 20 mg (test site) & & & \\
\hline$C_{\max }(n g / m L)$ & 175.42 & 175.56 & $8.8 \%$ & $100.1 \%$ & $96.2-104.1$ \\
\hline $\mathrm{AUC}_{0-12 \mathrm{~h}}(\mathrm{~h} \times \mathrm{ng} / \mathrm{mL})$ & 905.05 & 933.37 & $10.9 \%$ & $103.1 \%$ & $98.3-108.2$ \\
\hline $\mathrm{AUC}_{\text {last }}(\mathrm{h} \times \mathrm{ng} / \mathrm{mL})$ & 1088.35 & 1113.89 & $11.4 \%$ & $102.3 \%$ & $97.3-107.7$ \\
\hline
\end{tabular}




\section{Adverse events}

DR-HC at all doses was generally well tolerated. AEs were reported in 3/31 patients (10\%); all AEs were mild and none were considered by the investigator to be related to study drug. Two subjects reported mild intermittent hiccups considered by the investigator to be possibly related to dexamethasone. Two subjects experienced mild AEs (loose stools, abrasion) that were not considered to be treatment emergent. There were no deaths, serious AEs, or discontinuations due to an AE. There were no clinically relevant changes in laboratory parameters, vital signs, or ECGs.

\section{Discussion}

The aim of glucocorticoid replacement therapy for patients with AI is to mimic endogenous cortisol secretion as closely as possible in healthy subjects in terms of both total exposure and the diurnal cortisol exposure profile (21). Healthy individuals have a very low or undetectable level of cortisol at midnight, which gradually increases to the maximal concentration in the early morning and then declines over the rest of the day $(22,23)$. Unfortunately, conventional replacement therapy with immediaterelease hydrocortisone neither restores nor emulates the intrinsic circadian rhythm of cortisol secretion $(1,24)$. Current treatment regimens are associated with over- or underexposure of glucocorticoids and the multiple daily dosing required is often associated with poor patient adherence $(25,26)$.

The novel once-daily, dual-release oral formulation of hydrocortisone investigated in this study was developed to ensure high and robust bioavailability following absorption from the small intestine and proximal colon, thereby providing a predictable cortisol exposure profile over $24 \mathrm{~h}$ (1). The immediate-release component in the tablet coating provides a rapid rise in cortisol levels after morning oral intake when physiological levels are higher, while the hydrocortisone in the extended-release core is released more slowly so that cortisol concentrations are low when there is low physiological exposure and there is a cortisol-free interval at night, thereby avoiding dose accumulation with repeated doses (1).

Our findings show that once-daily DR-HC provides high exposure during the first $4 \mathrm{~h}$, reducing over the remainder of the day and a cortisol-free period during the night. The dual-release tablet therefore mimicked most phases of the physiological cortisol profile, with the exception of the early morning increase that occurs during sleep $(22,23)$. Our results support those from earlier clinical trials, in which peak plasma cortisol concentrations were observed for the first $4-6 \mathrm{~h}$ after dosing, with mean $\mathrm{T}_{\max }$ ranging from 0.63 to $1.11 \mathrm{~h}$ with the 5 and $20 \mathrm{mg}$ doses, followed by a gradual decrease to levels below $50 \mathrm{nmol} / \mathrm{L}(18 \mathrm{ng} / \mathrm{mL}) 18-24 \mathrm{~h}$ post-dose $(15,16)$. Furthermore, individual PK parameters for the 5 and $20 \mathrm{mg}$ doses evaluated in this study were generally similar to those reported in another PK study in healthy volunteers (15). DR-HC was well tolerated with no safety concerns after single dosing in Caucasian and Japanese healthy volunteers.

Treatment with conventional thrice-daily hydrocortisone is associated with exposure to high cortisol levels in the late afternoon and evening, which may lead to the development of increased cardiovascular risk (10, $12,27,28)$, disturbance of sleep pattern, and cognitive dysfunction $(14,29,30,31)$. By contrast, the afternoon and evening cortisol exposure profile with DR-HC in our study appeared to be similar to the endogenous cortisol profile and, in a previous clinical study in patients with AI, cortisol plasma concentrations following DR-HC declined during the afternoon to significantly reduced levels compared with conventional immediate-release hydrocortisone therapy given thrice daily (16). Hence, the multiple peaks and troughs in cortisol concentration that occur with conventional hydrocortisone therapy are avoided with DR-HC. The beneficial effects of the time-exposure profile of DR-HC have been demonstrated by significantly improved cardiovascular risk factors and metabolic disturbances compared with hydrocortisone three times per day, as well as significant improvements in patient QoL (16).

Studies investigating normal cortisol secretion in healthy children and adolescents $(n=14)$ and adults ( $n=33$ and $n=29$ ) have shown that there is a cortisolfree period at night, with cortisol concentrations tending to reach nadir of $<50-331 \mathrm{nmol} / \mathrm{L}(<18-119 \mathrm{ng} / \mathrm{mL})$ at around midnight $(20,32,33)$. Achieving this nighttime cortisol-free period is important from a safety aspect as it allows for repeated dosing without dose accumulation and helps to avoid the detrimental effects of increased nighttime cortisol levels $(10,11,13,14)$. Hence, the target cortisol plasma concentration at midnight in patients receiving glucocorticoid replacement therapy should be similarly low to optimize the clinical outcomes in this population. This is achieved with conventional thrice-daily hydrocortisone therapy (16), and this study indicates that this may also be achievable with once-daily DR-HC. Cortisol plasma concentrations of $0-65.0 \mathrm{ng} / \mathrm{mL}$ 
were obtained from the last evaluation of the day at 23:00h, which is in agreement with midnight serum cortisol levels in clinical studies in healthy volunteers $(20,32,33)$.

Treatment with DR-HC $20 \mathrm{mg}$ seemed to provide higher than endogenous cortisol plasma concentrations during the morning. In the earlier study conducted in AI patients, cortisol exposure during the first $4 \mathrm{~h}$ postdose with DR-HC was significantly higher than with conventional thrice-daily hydrocortisone therapy, whereas afternoon, evening, and overall exposure levels were significantly lower (16). Evidence suggests that the detrimental metabolic impact of high plasma cortisol levels in the morning may be minimal, whereas cortisol overexposure in the evening induces a state of insulin resistance and reduced insulin clearance (10). It is thought that the varying effects of morning and evening cortisol overexposure may be related to differences in cortisol clearance and glucocorticoid receptor regulation (10).

Within-subject, day-to-day PK variability in cortisol exposure with DR-HC was less than $15 \%$, probably due to the mechanism of absorption in the gastrointestinal tract. A consistent exposure-time profile within individual patients is a particular advantage of the formulation in the maintenance of glucocorticoid replacement therapy, as it is indicative of its reliability and minimal risk for any absorption failure. As well as minimizing the day-to-day variability in cortisol-time exposure, once-daily dosing is likely to improve patient compliance with therapy compared with multiple-dose regimens (34).

Intersubject variability in both the endogenous cortisol plasma profiles and the cortisol plasma concentrations after hydrocortisone treatment was high in our study, corroborating previous findings (15). The use of a weight-based nomogram for immediate-release hydrocortisone has been shown to reduce intersubject variability in cortisol exposure, as body weight is the main clinical determinant in cortisol clearance rate (35). Hence, the intersubject variability in PK in our study may be at least partly attributed to the wide variation in body weight, mainly resulting from the inclusion of two female Japanese outliers with low body weight, while the majority of remaining subjects were male with markedly higher body weight. Although the nomogram presented by Mah et al. (35) cannot be used for DR-HC due to the difference in relative bioavailability versus immediate-release hydrocortisone, it seems rational that application of a similar weight-based nomogram may be useful when administering DR-HC to reduce variability in hydrocortisone exposure between patients, thereby minimizing under- or overtreatment. Ethnicity of the participants did not appear to affect the PK of DR-HC when differences in body weight were controlled for. However, the effects of body weight on the PK of this formulation need to be considered in particular ethnic populations as over- or underexposure to cortisol may occur.

Our study showed that PK exposure parameters for DR-HC were less than dose proportional in the fasted state, which confirms previous findings for DR-HC (15) and is in line with PK data for immediate-release and suspension formulations of hydrocortisone in the same dose range $(36,37)$. This important PK property of oral hydrocortisone makes it difficult to determine the optimal dosing regimen during intercurrent illness in AI, for which current management is inadequate $(1,7,9,38)$. The recommendation for managing non-severe episodes of intercurrent illness with DR-HC, that is, increasing dosing frequency from once to twice or thrice daily with intervals of $8 \pm 2 \mathrm{~h}$ (i.e. not increasing the morning dose) (39), appears to provide greater cortisol exposure over $24 \mathrm{~h}$ compared with conventional immediate-release hydrocortisone thrice daily (40). This strategy helps to eliminate the less than dose-proportional exposure seen with increasing doses of hydrocortisone when administered as a single dose.

Plasma cortisol concentrations were measured using LC-MS/MS, a state-of-the-art methodology specific to cortisol (41). Published data on normal serum cortisol profiles are generally based on immunoassays, which lack the selectivity to fully differentiate between cortisol and formed cortisol metabolites. Consequently, plasma cortisol concentrations from immunoassays are higher than those from more selective analytical approaches, such as LC-MS/MS (15). This is an important consideration when comparing the cortisol concentrations reported here with previous data.

In summary, once-daily DR-HC demonstrates a plasma cortisol exposure profile that resembles the normal endogenous cortisol profile for the majority of the day. After a period of high exposure during the first $4 \mathrm{~h}$ post-dose, there is a gradual reduction in cortisol levels over the afternoon followed by a cortisol-free period at night. As well as confirming previous findings, this study has expanded the PK data to an additional dose and into a different ethnic group, and has demonstrated the low within-subject day-to-day variability of DR-HC, indicating a potential for improved safety versus conventional glucocorticoid replacement therapy. This study has also reaffirmed previous observations that cortisol exposure is less than dose proportional with increasing doses of hydrocortisone, which needs to be considered when 
managing intercurrent illness in AI. This new formulation of hydrocortisone warrants further investigation in clinical trials in patients with AI.

\section{Declaration of interest}

G J, H L, and S S have financial interests in Plenadren. G J and H L have acted as Consultants for Viropharma/Shire. G J has received lecture fees from Pfizer, Novo Nordisk, and Otsuka, and has also acted as a Consultant for AstraZeneca. C M is an employee at Shire International $\mathrm{GmbH}$, Switzerland, and K R is an employee at Shire PLC, USA.

Funding

This study was funded by Viropharma (now Shire).

\section{Acknowledgments}

The authors would like to thank the study personnel for their skilful support during this study and the subjects who volunteered to participate. Pharmacokinetic analysis was performed by James A Dowell, PhD, Principal, Pharmacology Development Services, LLC. Medical writing support for this manuscript was provided by Tamsin Williamson, and funded by Shire.

\section{References}

1 Johannsson G, Skrtic S, Lennernäs H, Quinkler M \& Stewart PM. Improving outcomes in patients with adrenal insufficiency: a review of current and future treatments. Current Medical Research and Opinion 201430 1833-1847. (doi:10.1185/03007995.2014.925865)

2 Arlt W \& Allolio B. Adrenal insufficiency. Lancet 2003361 18811893. (doi:10.1016/S0140-6736(03)13492-7)

3 Johannsson G, Falorni A, Skrtic S, Lennernäs H, Quinkler M, Monson JP \& Stewart PM. Adrenal insufficiency: review of clinical outcomes with current glucocorticoid replacement therapy. Clinical Endocrinology 201582 2-11. (doi:10.1111/cen.2014.82.issue-1)

4 Bensing S, Brandt L, Tabaroj F, Sjöberg O, Nilsson B, Ekbom A, Blomqvist P \& Kämpe O. Increased death risk and altered cancer incidence pattern in patients with isolated or combined autoimmune primary adrenocortical insufficiency. Clinical Endocrinology 200869 697-704. (doi:10.1111/j.1365-2265.2008.03340.x)

5 Bergthorsdottir R, Leonsson-Zachrisson M, Odén A \& Johannsson G. Premature mortality in patients with Addison's disease: a populationbased study. Journal of Clinical Endocrinology and Metabolism 200691 4849-4853. (doi:10.1210/jc.2006-0076)

6 Bleicken B, Hahner S, Loeffler M, Ventz M, Decker O, Allolio B \& Quinkler M. Influence of hydrocortisone dosage scheme on health-related quality of life in patients with adrenal insufficiency. Clinical Endocrinology 201072 297-304. (doi:10.1111/j.13652265.2009.03596.x)

7 Burman P, Mattsson AF, Johannsson G, Höybye C, Holmer H, Dahlqvist P, Berinder K, Engström BE, Ekman B, Erfurth EM et al. Deaths among adult patients with hypopituitarism: hypocortisolism during acute stress, and de novo malignant brain tumors contribute to an increased mortality. Journal of Clinical Endocrinology and Metabolism 201398 1466-1475. (doi:10.1210/jc.2012-4059)

8 Tomlinson JW, Holden N, Hills RK, Wheatley K, Clayton RN, Bates AS, Sheppard MC \& Stewart PM. Association between premature mortality and hypopituitarism. West Midlands Prospective
Hypopituitary Study Group. Lancet 2001357 425-431. (doi:10.1016/ S0140-6736(00)04006-X)

9 Hahner S, Spinnler C, Fassnacht M, Burger-Stritt S, Lang K, Milovanovic D, Beuschlein F, Willenberg HS, Quinkler M \& Allolio B. High incidence of adrenal crisis in educated patients with chronic adrenal insufficiency: a prospective study. Journal of Clinical Endocrinology and Metabolism 2015100 407-416. (doi:10.1210/ jc.2014-3191)

10 Plat L, Leproult R, L'Hermite-Baleriaux M, Fery F, Mockel J, Polonsky KS \& Van Cauter E. Metabolic effects of short-term elevations of plasma cortisol are more pronounced in the evening than in the morning. Journal of Clinical Endocrinology and Metabolism 199984 3082-3092. (doi:10.1210/jcem.84.9.5978)

11 Kumari M, Badrick E, Ferrie J, Perski A, Marmot M \& Chandola T. Self-reported sleep duration and sleep disturbance are independently associated with cortisol secretion in the Whitehall II study. Journal of Clinical Endocrinology and Metabolism 200994 4801-4809. (doi:10.1210/jc.2009-0555)

12 Filipsson H, Monson JP, Koltowska-Häggström M, Mattsson A \& Johannsson G. The impact of glucocorticoid replacement regimens on metabolic outcome and comorbidity in hypopituitary patients. Journal of Clinical Endocrinology and Metabolism 200691 3954-3961. (doi:10.1210/jc.2006-0524)

13 Scheer FA, Hilton MF, Mantzoros CS \& Shea SA. Adverse metabolic and cardiovascular consequences of circadian misalignment. PNAS 2009106 4453-4458. (doi:10.1073/pnas.0808180106)

14 Vgontzas AN, Bixler EO, Lin HM, Prolo P, Mastorakos G, Vela-Bueno A, Kales A \& Chrousos GP. Chronic insomnia is associated with nyctohemeral activation of the hypothalamic-pituitary-adrenal axis: clinical implications. Journal of Clinical Endocrinology and Metabolism 200186 3787-3794. (doi:10.1210/jcem.86.8.7778)

15 Johannsson G, Bergthorsdottir R, Nilsson AG, Lennernas H, Hedner T \& Skrtic S. Improving glucocorticoid replacement therapy using a novel modified-release hydrocortisone tablet: a pharmacokinetic study. European Journal of Endocrinology 2009161 119-130. (doi:10.1530/EJE-09-0170)

16 Johannsson G, Nilsson AG, Bergthorsdottir R, Burman P, Dahlqvist P, Ekman B, Engström BE, Olsson T, Ragnarsson O, Ryberg M et al. Improved cortisol exposure-time profile and outcome in patients with adrenal insufficiency: a prospective randomized trial of a novel hydrocortisone dual-release formulation. Journal of Clinical Endocrinology and Metabolism 201297 473-481. (doi:10.1210/jc.20111926)

17 Quinkler M, Miodini Nilsen R, Zopf K, Ventz M \& Øksnes M. Modified-release hydrocortisone decreases BMI and HbA1c in patients with primary and secondary adrenal insufficiency. European Journal of Endocrinology 2015172 619-626. (doi:10.1530/EJE-14-1114)

18 Zimmerman M \& Coryell W. The dexamethasone suppression test in healthy controls. Psychoneuroendocrinology 198712 245-251. (doi:10.1016/0306-4530(87)90048-5)

19 FDA Guidance for Industry 2003. Bioavailability and bioequivalence studies submitted in NDAs or INDs-general consideration (available at: http://www.fda.gov/Drugs/ GuidanceComplianceRegulatoryInformation/Guidances/default.htm) (accessed: August 2014).

20 Schuirmann DJ. A comparison of the two one-sided tests procedure and the power approach for assessing the equivalence of average bioavailability. Journal of Pharmacokinetics and Biopharmaceutics 1987 15 657-680. (doi:10.1007/BF01068419)

21 de Miguel Novoa P, Vela ET, García NP, Rodríguez MM, Guerras IS Martínez de Salinas Santamaría Mde L, Masó AA \& Área de Conocimiento del Grupo de Neuroendocrino de la SEEN (Grupo Insuficiencia Adrenal). Guidelines for the diagnosis and treatment of adrenal insufficiency in the adult. Endocrinología y Nutrición 201461 (Supplement 1) 1-35. (doi:10.1016/S1575-0922(14)73526-0) 
22 Debono M, Ghobadi C, Rostami-Hodjegan A, Huatan H, Campbell MJ, Newell-Price J, Darzy K, Merke DP, Arlt W \& Ross RJ. Modified-release hydrocortisone to provide circadian cortisol profiles. Journal of Clinical Endocrinology and Metabolism 200994 1548-1554. (doi:10.1210/jc.2008-2380)

23 Weitzman ED, Fukushima D, Nogeire C, Roffwarg H, Gallagher TF \& Hellman L. Twenty-four hour pattern of the episodic secretion of cortisol in normal subjects. Journal of Clinical Endocrinology and Metabolism 197133 14-22. (doi:10.1210/jcem-33-1-14)

24 Simon N, Castinetti F, Ouliac F, Lesavre N, Brue T \& Oliver C. Pharmacokinetic evidence for suboptimal treatment of adrenal insufficiency with currently available hydrocortisone tablets. Clinical Pharmacokinetics 201049 455-463. (doi:10.2165/11531290000000000-00000)

25 Chan S \& Debono M. Replication of cortisol circadian rhythm: new advances in hydrocortisone replacement therapy. Therapeutic Advances in Endocrinology and Metabolism 20101 129-138. (doi:10.1177/2042018810380214)

26 Debono M, Ross RJ \& Newell-Price J. Inadequacies of glucocorticoid replacement and improvements by physiological circadian therapy. European Journal of Endocrinology 2009160 719-729. (doi:10.1530/EJE08-0874)

27 Matthews K, Schwartz J, Cohen S \& Seeman T. Diurnal cortisol decline is related to coronary calcification: CARDIA study. Psychosomatic Medicine 200668 657-661. (doi:10.1097/01. psy.0000244071.42939.0e)

28 Danilowicz K, Bruno OD, Manavela M, Gomez RM \& Barkan A. Correction of cortisol overreplacement ameliorates morbidities in patients with hypopituitarism: a pilot study. Pituitary $2008 \mathbf{1 1}$ 279-285. (doi:10.1007/s11102-008-0126-2)

29 Steiger A \& Holsboer F. Neuropeptides and human sleep. Sleep 1997 20 1038-1052.

30 Chrousos GP \& Gold PW. The concepts of stress and stress system disorders. Overview of physical and behavioral homeostasis. JAMA 1992267 1244-1252. Review. Erratum in: JAMA 1992 268 200. (doi:10.1001/jama.1992.03490020044029)

31 Späth-Schwalbe E, Uthgenannt D, Körting N, Fehm HL \& Born J. Sleep and wakefulness affect the responsiveness of the pituitary-adrenocortical axis to arginine vasopressin in humans. Neuroendocrinology 199460 544-548.
32 Wallace WH, Crowne EC, Shalet SM, Moore C, Gibson S, Littley MD \& White A. Episodic ACTH and cortisol secretion in normal children. Clinical Endocrinology 199134 215-221. (doi:10.1111/j.1365-2265.1991.tb00297.x)

33 Newell-Price J, Trainer P, Perry L, Wass J, Grossman A \& Besser M. A single sleeping midnight cortisol has $100 \%$ sensitivity for the diagnosis of Cushing's syndrome. Clinical Endocrinology 199543 545-550. (doi:10.1111/j.1365-2265.1995.tb02918.x)

34 Srivastava K, Arora A, Kataria A, Cappelleri JC, Sadosky A \& Peterson AM. Impact of reducing dosing frequency on adherence to oral therapies: a literature review and meta-analysis. Patient Preference and Adherence 20137 419-434. (doi:10.2147/PPA.S44646)

35 Mah PM, Jenkins RC, Rostami-Hodjegan A, Newell-Price J, Doane A, Ibbotson V, Tucker GT \& Ross RJ. Weight-related dosing, timing and monitoring hydrocortisone replacement therapy in patients with adrenal insufficiency. Clinical Endocrinology 200461 367-375. (doi:10.1111/j.1365-2265.2004.02106.x)

36 Toothaker RD, Sundaresan GM, Hunt JP, Goehl TJ, Rotenberg KS, Prasad VK, Craig WA \& Welling PG. Oral hydrocortisone pharmacokinetics: a comparison of fluorescence and ultraviolet highpressure liquid chromatographic assays for hydrocortisone in plasma. Journal of Pharmaceutical Sciences 198271 573-576. (doi:10.1002/ jps.2600710522)

37 Toothaker RD, Craig WA \& Welling PG. Effect of dose size on the pharmacokinetics of oral hydrocortisone suspension. Journal of Pharmaceutical Sciences 198271 1182-1185. (doi:10.1002/jps.2600711029)

38 Smans LC, Van der Valk ES, Hermus AR \& Zelissen PM. Incidence of adrenal crisis in patients with adrenal insufficiency. Clinical Endocrinology 201684 17-22. (doi:10.1111/cen.12865)

39 Plenadren modified-release tablets (hydrocortisone). Summary of Product Characteristics. ViroPharma SPRL. November 2011 (available at: http://www.ema.europa.eu/docs/en_GB/document_library/ EPAR_-_Product_Information/human/002185/WC500117637.pdf).

40 Simonsson USH, Johannsson G, Skrtic S, Lennerläs H \& Marelli C. Management of patients with Addison's disease using dual-release hydrocortisone during periods of intercurrent illness. Endocrine Abstracts 201332 P24. (doi:10.1530/endoabs.32.P24)

41 Turpeinen U \& Hämäläinen E. Determination of cortisol in serum, saliva and urine. Best Practice \& Research: Clinical Endocrinology \& Metabolism 201327 795-801. (doi:10.1016/j.beem.2013.10.008)

Received 29 September 2015

Revised version received 13 November 2015

Accepted 7 December 2015 